\title{
Enquête
}

Archives de la revue Enquête

$2 \mid 1995$

Usages de la tradition

\section{Tradition et identité}

La tradition dogon entre traditionalisme rural et néo-traditionalisme urbain

Dogon tradition between rural traditions and urban neo-traditionalism

Jacky Bouju

\section{(2) OpenEdition}

1 Journals

Édition électronique

URL : http://journals.openedition.org/enquete/313

DOI : $10.4000 /$ enquete.313

ISSN : 1953-809X

Éditeur :

Cercom, Éditions Parenthèses

Édition imprimée

Date de publication : 31 décembre 1995

Pagination : 95-117

Référence électronique

Jacky Bouju, «Tradition et identité », Enquête [En ligne], 2 | 1995, mis en ligne le 10 juillet 2013,

consulté le 18 avril 2019. URL : http://journals.openedition.org/enquete/313 ; DOI : 10.4000/

enquete. 313

Ce document a été généré automatiquement le 18 avril 2019 


\title{
Tradition et identité
}

\author{
La tradition dogon entre traditionalisme rural et néo-traditionalisme \\ urbain
}

Dogon tradition between rural traditions and urban neo-traditionalism

\section{Jacky Bouju}

Quand la mémoire va ramasser du bois mort, elle ramène le fagot qui lui plaît! Proverbe de la Boucle du Niger

1 Il y a peu de temps encore, l'étude de la tradition apparaissait, à tort ou à raison, comme la marque distinctive du métier d'ethnologue. Pourtant, de manière assez paradoxale, la question générale de sa caractérisation conceptuelle est restée passablement négligée par la théorie anthropologique. Cela tient sans doute au fait, que le mot tradition est un «mot-problème », c'est-à-dire, selon G. Lenclud, un «mot-outil » qui permet une procédure grossière d'identification sans connaissance approfondie du référent de ce mot, un mot qui "signale un concept » plutôt qu'il n'identifie un objet ou même qu'il n'exprime un sens. Qui plus est, un concept est nécessairement équivoque "puisqu'il réunit en lui une pluralité de significations ${ }^{1}$ ». Ceci étant dit, on peut considérer qu'une première signification du mot tradition renvoie à une chose, un énoncé ou une manière de faire (ou de dire) qui est l'objet d'une transmission². Une seconde crédite l'objet ainsi défini d'une valeur culturelle fondamentale. Enfin, une troisième conçoit la tradition comme une propriété de cet objet à savoir qu'il est transmis de génération en génération dans un but conservatoire.

2 J. Pouillon fut le premier anthropologue à conjuguer la tradition au présent. Dès 1975, il définissait la tradition comme une «rétro-projection» et, par là même, invalidait l'hypothèse essentielle de la " conservation » dont, aujourd'hui encore, le sens commun continue d'entretenir le préjugé. La tradition n'est pas ce qui a toujours été, elle n'est jamais un simple produit du passé mais plutôt une invention, " une interprétation du passé conduite en fonction de critères rigoureusement contemporains. [...] Nous choisissons ce par quoi nous nous déclarons déterminés, nous nous présentons comme les continuateurs de ceux dont nous avons fait nos prédécesseurs ${ }^{3}$.» Plus exactement, la 
tradition serait comme le fruit d'une «rétrospection camouflée »: «Les sociétés qui se disent modernes ne sont pas des sociétés qui se défont de leur passé: elles le réaménagent en fonction de leurs besoins présents. Comme, en outre, elles sont complexes et conflictuelles, plusieurs types de réaménagement sont en concurrence, et des contemporains s'affrontent par passés interposés en se choisissant leurs ancêtres [...] ce sont les fils qui engendrent leurs pères pour justifier les changements réels qu'ils apportent au système existant ${ }^{4}$.» Après lui, E. Hobsbawm puis G. Lenclud ${ }^{5}$ ont repris ce point de vue selon lequel la tradition est une « invention » du présent.

Ce point de vue n'est pas classique en ethnologie. Aussi a-t-on envie d'interroger cette discipline pour savoir si la polysémie de la notion de tradition, si particulière à notre sens commun et à notre culture, existe aussi dans d'autres cultures. Ce faisant, on constate, assez paradoxalement, que bien peu d'ethnologues s'intéressent à la conception de la tradition dans les sociétés soi-disant «traditionnelles " qu'ils ont généralement coutume d'étudier. Après J. Goody ${ }^{6}, \mathrm{P}$. Boyer fut l'un des premiers à se placer au point de vue de la tradition orale. En s'inscrivant dans la ligne interprétative tracée par le philosophe E. Weil ${ }^{7}$, il considère que la tradition n'existe que proclamée, « fabriquée » par le contexte « traditionnel » qui lui confère autorité 8 .

Dans cet essai, j'essaierai de montrer, en m'appuyant sur un strict examen de données ethnographiques originales, comment les Dogons karambé ${ }^{2}$ reçoivent leur tradition et la proclament. Le décentrement de perspective que permet d'opérer l'adoption de ce point de vue emic présente un intérêt heuristique certain. Je conjuguerai donc la tradition dogon au présent de sa «fabrication » et de son «invention ${ }^{10}$ " locales. D'abord, en me situant dans le contexte traditionaliste d'un conseil du clan, je montrerai que le mécanisme cognitif « traditionnel » mis en évidence par Pascal Boyer « traditionne ${ }^{11}$ » par transcendance. Ensuite, j'interrogerai le statut de la tradition en me fondant sur une analyse des conceptions que véhicule le dialecte donno soo des Dogons karambé. Pour finir, j'essaierai de distinguer le traditionalisme rural de la posture néo-traditionaliste qui est apparue récemment dans les milieux dogons de la capitale malienne.

\section{Le contexte de « fabrication » de la tradition}

5 La transcription ${ }^{12}$ qui sert de base à mon analyse a une petite histoire. Au cours du travail d'analyse des données consécutif à mon premier séjour à Bandiagara (en 1979-1980), j'avais éprouvé le besoin de disposer d'informations supplémentaires touchant à l'histoire du groupe et aux cultes locaux. J'avais expliqué mes intentions et présenté mes questions à A. Karambé, l'homme le plus âgé du quartier fondateur du village de Sibi-Sibi et, à ce titre, celui qui m'avait accordé l'hospitalité et décidé de mon lieu précis de résidence au village. Il me fit comprendre que le thème ou la nature de certaines questions étaient tels qu'on ne pourrait pas s'en tenir à l'un de nos habituels entretiens ethnographiques ${ }^{13}$. J'en restais donc là pendant qu'il se concertait avec les responsables des autres villages karambés. La concertation prit tout le temps nécessaire à l'explicitation de mes intentions et c'est au cours de la saison sèche de l'année 1981 (alors que j'étais définitivement rentré en France) qu'un courrier m'apprit que les Karambés avaient pris la décision de réunir « l'assemblée du clan » togu mondu pour proclamer la tradition et que la séance pourrait être enregistrée par un ami dogon. Le village de Dyombolo où résidait le dignitaire le plus âgé du clan ${ }^{14}$ était, pour cette raison, le lieu obligé de l'événement. Les membres du conseil comptaient les dignitaires religieux ainsi que tous les chefs de quartiers 
accompagnés de leurs assistants ${ }^{15}$ : en tout, douze hommes. L'ensemble de cette procédure produisait un contexte d'énonciation ${ }^{16}$ qui constituait la condition nécessaire à la proclamation de la tradition des Dogons karambé ${ }^{17}$.

Mais pour comprendre ce qui était précisément en jeu dans cet événement local, il convient de le mettre en rapport avec le contexte global de son occurrence. La religion musulmane est présente, au moins depuis le $\mathrm{xVI}^{\mathrm{e}}$ siècle, dans l'environnement immédiat de la culture dogon ${ }^{18}$. Les peuples qui vivent autour des Dogons (Bozos, Markas, Malinkés, Djénnenkés, Touaregs) et même au milieu d'eux (Peuls, gens de caste, colporteurs yarsés, dyoulas, hausas, etc.) sont, depuis longtemps, majoritairement musulmans. Surtout, dans la plupart des villages, aujourd'hui presque tous les jeunes gens se disent musulmans. Par ailleurs, le christianisme existe à travers quelques missions catholiques et protestantes qui ont une influence locale extrêmement limitée.

7 Au plan politique, on observe une situation analogue. Dans la deuxième moitié du XIX siècle, quelque temps avant l'arrivée des Français, les Dogons avaient subi la domination de l'empire théocratique des Peuls du Macina avant de tomber sous le joug de la dynastie toucouleur du royaume de Bandiagara. La colonisation française allait soumettre ce royaume et administrer la région dogon jusqu'à l'indépendance, où elle passe le pouvoir à l'État malien. Les Dogons ont donc, comme tous les autres peuples africains, une expérience, à la fois ancienne et variée, de la domination politique par une puissance extérieure. À l'époque de cet enregistrement, le pouvoir politique officiel était confisqué par l'Union démocratique du Peuple malien, le parti unique qui avait des délégués dans chaque quartier de chaque village du pays. De son point de vue et de celui de l'administration d'État, le clan était toléré comme une "survivance " historique, une structure coutumière destinée à disparaître. Dans les faits, cette « survivance » illégale se reproduisit malgré tout dans les vides et les replis de l'administration officielle qui s'arrangeait quotidiennement avec elle.

8 C'est donc dans ce contexte historique que le conseil du clan karambé s'est réuni. À l'évidence, un tel contexte n'est pas traditionnel au sens défini par E. Weil: il est traditionaliste. En effet, si le traditionalisme présuppose d'un côté une représentation consciente de ce qui est censé constituer l'héritage culturel, et de l'autre une comparaison avec d'autres choix possibles débouchant sur le choix de la tradition, alors la société dogon est, depuis longtemps déjà, bien installée dans le traditionalisme. Celui-ci apparaît dans ce contexte comme la seule posture possible de celui qui est légitimement placé en position d'héritier de la « tradition » et qui accepte cette place ${ }^{19}$.

\section{La tradition transcrite}

(Le doyen du clan A. Karambé est introduit par un cadet qui le désigne à l'assemblée et aux ancêtres comme celui qui peut et qui va proclamer la vérité de la tradition :)

1 « Le voici, c'est lui qui va dire, c'est lui qui va dire, c'est lui qui va dire. »

2 (A. Karambé :) « Nous avons bien passé la nuit, que la journée soit bonne.

3 Choses qui volent, choses qui rampent,

4 dieux d'en haut ${ }^{20}$, nous avons bien passé la nuit

5 dieux d'en bas $^{21}$, nous avons bien passé la nuit ;

6 nous avons eu une bonne journée,

7 donnez-nous un bon jour...

8 C'est le "Dieu céleste" amba ${ }^{22}$ qui est appelé ;

9 si le Dieu céleste a été appelé, c'est la "Déesse terre" Yayye qui est appelée 
10 Terre-nourricière au ventre gravide, notre Maîtresse toute puissante ;

11 nous avons eu du mil, donne-nous une bonne journée,

12 nous prions pour demander de bonnes choses, donne-nous la paix-santé,

13 lêbê Guindo ${ }^{23}$, nous avons vu l'aube,

14 ba-binu ${ }^{24}$, aux huit terrasses de pouvoir,

15 (grâce à) vous deux ensemble nous avons vu le jour ;

16 apportez-nous un corps en bonne santé,

17 apportez-nous une longue vie et des lendemains meilleurs,

18 apportez-nous la bonne parole,

19 détruisez les sorciers (car) nous sommes installés en paix.

$20 \mathrm{Kolumba}^{25}$ a fondé le pays (répété trois fois) et nous avons vu le jour,

21 sur son trône nous sommes assis, salut du matin!

22 Nous sommes les petits-enfants, notre langue ne doit pas glisser,

23 c'est sur votre trône que nous sommes assis, à toi parle ! »

(A. Karambé, son cadet prend la parole :)

24 « Samolu kindê, c'est l'aube

25 sur ton trône nous sommes assis,

26 conduis-nous en te mettant à notre tête et parle pour raconter

27 Antandu sin dongu, c'est l'aube

28 sur ton trône nous sommes assis ;

29 nous avons eu de bons jours, salut du matin!

30 Aujourd'hui nous n'avons rien créé qui soit différent,

31 nous sommes les petits-enfants,

32 les petits-enfants que vous avez laissés.

33 Même si nous sommes (bien) installés,

34 nous ne sommes pas avec une autre vision du monde qui soit différente.

35 Le serpent n'a pas peur du nid de sa mère :

36 il entre dans le nid de sa mère ${ }^{26}$.

37 Nous n'avons pas quitté le territoire, nous n'avons pas quitté le territoire.

38 Vous avez fait du bien le matin et vous avez fait du bien le soir.

39 Duru Antimbe-semi, Antandu Sin-dongu c'est vous qui avez fondé le territoire,

40 Konsogu Lagabêdê apportez-nous la bonne parole ;

41 autrefois, c'est eux, qui ont partagé les territoires,

42 qu'ils nous apportent la bonne parole ;

43 nous sommes venus en paix et en bonne santé,

44 nous partirons en paix et en bonne santé.

45 Autrefois, les gens d'Ebêgêlê avaient fondé un territoire,

46 quand nous sommes venus nous les avons trouvés,

47 autrefois, les gens d'avant les Dogons avaient fondé un territoire

48 quand nous sommes venus nous les avons trouvés ;

49 autrefois, quand les Tarawele de Montegiru sont venus ils nous ont trouvés, 50 c'est eux tous qui ont partagé l'espace et notre territoire fut créé ;

51 c'est avec nous que le territoire fut créé.

52 Quand nous sommes venus, nous n'avons rien trouvé

53 nous, nous n'avons rencontré personne.

54 C'est vous tous (les ancêtres fondateurs) qui portez la responsabilité de tout cela, 55 nous, ces choses nous ne les connaissons pas,

56 nous n'avons pas vu les choses d'autrefois,

57 nous disons ce qui a été dit.

58 Si ce n'est pas cela, nous n'avons pas connaissance de ces choses-là.

59 En nous appuyant sur ce qui vient d'être dit, nous allons palabrer

60 La bonne connaissance ${ }^{27}$ qui est apparue partout en ce lieu a été créée par nous

61 le mal, tout ce qui est mauvais a été chassé et mis en brousse.

62 Donnez-nous un corps sain, donnez-nous une longue vie

63 [...] Notre langue ne doit pas glisser ;

64 Voilà comment l'histoire est venue :» 
(Plusieurs thèmes sont alors successivement abordés: l'histoire du peuplement du territoire, l'ordre d'arrivée des ancêtres des clans voisins, l'abandon du village d'origine sous la pression des Toucouleurs à la fin du xix ${ }^{e}$ siècle.)

65 «... Ce n'est pas nous qui disons, ce sont nos ancêtres qui ont dit,

66 nous, nous sommes les petits-enfants.

67 Ils ont dit qu'ils se sont installés parce qu'ils n'ont rencontré personne

68 et c'est après eux que sont venus les villages suivants ${ }^{28}$. "

(Le narrateur se met à décliner l'ordre de fondation des villages dans leur territoire.)

69 «... C'est ça que nous avons entendu et compris »

(Puis, on évoque de nouveau les droits anciens qui confèrent au Karambé le pouvoir rituel sur leur territoire.)

70 « ... Certes, c'est cela qui nous a été dit, nous ne savons rien d'autre. »

71 (S. K. :) « Bon! ça va comme ça, on va appeler le nom des vieux qui sont assis ici :

72 Père Am. ? (cher du quartier ginna-pay du village de Dyombolo, lêbê dugo-no du

clan karambé) (à quoi l'assistance répond :) il est là

73 Père A. ? (chef du quartier ginna-pay du village de Sibi-Sibi) il est là

74 Père A. ? (Chef du quartier ginna-day du village de Dyombolo) il est là "

À ce moment, le locuteur se répète et il est immédiatement corrigé par l'assistance.

75 B. ? (Assistant du précédent, quartier ginna-day du village de Dyombolo) il est là

76 A. ? (Successeur du chef du quartier ginna-day du village de Sibi-Sibi) il est là

77 A. ? (Assistant du précédent, quartier ginna-day du village de Sibi-Sibi) il est là

78 A. ? (Assistant du chef du quartier ginna-pay du village de Dyombolo) il est là

79 D. ? (Chef du quartier ginna-tombolo du village de Kolountanga) il est là

$80 \mathrm{~A}$. ? (Chef du quartier ginna-pay du village de Kolountanga) il est là

$81 \mathrm{~S}$. ? (Chef du village de Sibi-Sibi, quartier ginna-pay du village de Sibi-Sibi) il est là

82 U. ? (Chef du village de Dyombolo, quartier ginna-day du village de Dyombolo) il

est là

83 M. ? (Kezu quartier ginna-binê du village de Kolountanga) il est là

84 « Nos anciens se sont réunis, à Dyombolo, ils se sont assis

85 pour raconter la tradition ${ }^{29}$ d'autrefois

86 [...] C'est cela la limite de ce qu'il nous est donné de dire.

87 Je dis mon recours en Dieu et en lui seul. »

10 (Après cette formule qui clôt la première partie du conseil où fut énoncée la tradition historique, commence la palabre. Maintenant, en réponse aux questions posées, les membres du conseil procèdent à la narration d'événements particuliers de l'histoire du clan ou développent des anecdotes renvoyant aux événements déjà évoqués tels que l'itinéraire migratoire depuis le Mande originel ${ }^{30}$, la guerre de Douna-pey avec les autres clans dogons, l'arrivée dans la région actuelle et l'installation du chasseur Antandu Sin Dongu, la confrontation avec les chasseurs-fondateurs des clans voisins, l'appropriation $\mathrm{du}$ territoire et le traçage des frontières par le feu, l'arrivée des immigrants à qui les Karambé ont donné une terre, la description des limites du territoire dont le locuteur affirme "la vérité », la donation de droits de culture aux immigrants, le retour sur les détails de la guerre de Douna-pey, la mention des puissances qui ont dominé la région au XIX ${ }^{e}$ siècle ; l'évocation de la puissance du royaume de Ségou à l'occasion de laquelle une autre personne prend la parole pour mentionner l'acquisition du culte «mosquée de Ségou " Segu minjiru lors de l'étape à Ségou Sikoro pendant la migration du Mande, l'énumération de tous les villages karambé connus, la devise du clan et son rapport aux grandes devises dogons, pour terminer avec des détails sur le peuplement de la région. À la suite d'une question trop précise, le narrateur rétorque :) 
88 «... Nous ne savons pas, car nous sommes des enfants,

89 ces choses (que nous tenons) de la bouche de nos ancêtres,

90 nous ne les comprenons plus, personne ne peut plus en parler... »

11 (La palabre continue avec l'évocation des raids esclavagistes menés par l'empire peul du Macina et de l'aide apportée par les Dogons aux troupes d'El Hadj Omar Tall. Après quoi le locuteur apporte de nouvelles précisions sur l'étendue du territoire peuplé par les Karambé, la description de leur maîtrise rituelle sur la terre, et enfin les règles d'octroi de droit de culture aux étrangers.)

91 «Alors! dé J. ${ }^{31}$ a bien dit tous les détails n'est-ce pas ? ...»

12 (Le vieillard pense sans doute en avoir omis quelques-uns car il reprend le thème des devises des clans et celui de leur ordre d'arrivée dans la région. Puis, S. prend la parole pour raconter l'épisode de l'arrivée des réfugiés de Sokolo. Après quoi, délaissant les événements et anecdotes historiques on aborda les questions religieuses. La première question qui portait sur les cultes du serpent associés au binu et au lèbè rencontra une certaine réticence que rompit kezu M. en racontant la légende du génie d'eau. Mais à la question de savoir ce qu'étaient le ba-binu et l'origine de son culte, les problèmes surgirent :)

92 (S. :) « Si cette connaissance est affaiblie,

93 "chose 32 " peut écraser la vie de quelqu'un ;

94 personne ne peut parler de ce qui est plus grand que sa bouche ;

95 même si nous savons, on ne peut parler de ce qui est plus grand que la bouche

96 celui qui porte le collier du ba-binu est assis ici

97 mais il ne peut pas parler de ça.

98 Nous savons comment Dieu nous a donné le ba-binu

99 mais on ne peut pas en parler ;

100 il est certain que nous ici, tout autant que lui, nous ne pouvons pas en parler ;

101 il faut passer sur ce don de Dieu, on ne peut rien dire d'autre

102 car ce ba-binu est devenu très puissant. [...]»

(Malgré ces injonctions, le serviteur du culte du ba-binu, kezu M. commence à décrire certains aspects du culte. Mais il est presque immédiatement arrêté par l'assistance :)

103 « kezu il faut arrêter là !»

(Toutefois, le dignitaire continue à parler, transgressant volontairement l'interdit de dévoiler qu'on lui rappelle avec insistance :)

104 (les allocutaires :) «Réparation ! réparation ! ...»

105 (kezu M. :) « Maintenant, en excuse, je confie la brousse à Dieu... »

106 (l'assistance :) « kezu ! on ne peut pas entendre ceci !

107 L'endroit d'où est sorti le ba-binu et pourquoi, ça ne se dit pas!

108 On ne peut parler de ce qui est plus grand que la bouche !»

109 (S. :) « Maintenant, si tu laisses ce sujet, laisse ainsi, sans en dire plus ! »

(Le kezu continue imperturbablement. Alors, S. prend la parole pour assumer la parole interdite $^{33}$ à la place du kezu et empêcher la propagation de l'impureté rituelle consécutive au sacrilège :)

110 (S. :) « Maintenant, je demande pardon,

111 une personne de notre clan a beaucoup vieilli

112 elle a vieilli au-delà de ce qui est imaginable et elle ne peut plus rien manger

113 alors, cette personne est devenue "serpent" n'nà

114 si elle s'est changée en serpent (alors) ce serpent-là c'est la fécondité du clan, il

ne faut pas le tuer!

115 voilà, ce qu'il en est (du ba-binu), c'est ainsi, il ne faut pas parler de ce qui a été

dit,

116 nous ne devons pas parler de ce qui a été dit,

117 si en racontant nous avons eu l'audace de parler de lui, 
118 maintenant, il ne faut plus en parler ;

119 et maintenant, il y a lui ${ }^{34}$ qui est ici et ce dont il a parlé,

120 le jour du sacrifice au binu, à cause de tout ce qu'il a dit avec sa propre bouche

121 lui, le jour du sacrifice au binu, qu'il soit pardonné ;

122 à cause de ça, il donnera du bétail, il donnera des poulets

123 car lui ne doit pas parler de ces choses-là. »

(Malgré cette « mise à l'amende », kezu M. continue de dévoiler le mystère du ba-binu :)

124 « Si on considère le vrai ba-binu, le véritable dont on vient de parler

125 ba-binu et la personne humaine ne font qu'un, c'est le seul ba-binu

126 [...] le secret de ba-binu c'est nous; nous sommes la progéniture de ba-binu

127 Dieu a créé l'être humain (mais) le secret de la progéniture de ba-binu c'est

nous :

128 les Karambé, les Hogons de Dyon avec cette terre-nourricière.

129 Sur cette terre et au ciel, l'aîné de Dyon (dyon badyî) c'est lui notre Dieu amba.

130 Depuis le temps du Mande, le fils aîné ayant été créé, il est venu par ici.

131 [...] Si on veut bien voir ba-binu est une personne humaine

132 et nous, nous sommes les enfants de ba-binu

133 le serpent yuguru est "sorti" de notre ancêtre Dom-bado (dyon badyî) 13,4 [...] il

n'y a pas de différence entre ba-binu et la personne humaine.

135 [...] Certaines personnes pratiquent le culte de ce serpent mais les gens

136 des clans différents n'ont pas besoin de respecter cet interdit.

137 Nous par contre, nous offrons des sacrifices car nous avons besoin de pratiquer, sinon...»

(De nouveau, Sodyougo interrompt le kezu pour prendre sa place de narrateur :)

138 «Ce dont nous venons de parler,

139 nous a fait dépasser la limite de ce que nous pouvions dire

140 ce serpent n'nà c'est notre personne qui s'est changée en serpent

141 oui, alors qu'elle buvait, le serpent a pénétré sa personne en entrant par sa bouche

142 et l'eau ainsi bue l'a "déchirée" karambe. Et maintenant cette devise est la nôtre : karambé,

143 le serpent n'nà est notre interdit, c'est notre ba-binu

144 et notre nom-devise à tous est "les déchirés" karambe

145 mais on n'aime pas beaucoup parler de cela... »

\section{Tradition, invention, conformisme}

Le concept de tradition apparaît dans le texte dans l'expression ya atembi soo-go «la parole d'autrefois que l'on a trouvée » (1.85). Cette expression montre que le dialecte dogon donno distingue entre deux connotations : la tradition comme « héritage » atembi et la tradition comme «message » soo-go. En effet, ya atembi - littéralement ${ }^{35}$ « ce que l'on a trouvé du passé » - désigne « la tradition » dans tous les dialectes dogons. «Ce que l'on a trouvé » c'est ce qui se disait et se faisait et qu'on a pu entendre et voir : la tradition est un savoir appris en écoutant les anciens parler et en les regardant faire. Mais il ne suffit pas de «trouver » une tradition encore faut-il la « recevoir » afin que ce que l'on a trouvé constitue un héritage ${ }^{36}$. Il est important, je crois, de souligner le fait que la notion dogon de tradition connote cette conception, fort répandue, de la tradition comme héritage culturel.

Il me parait plus intéressant encore de noter les différences de conception que masque cette similitude apparente. On remarque alors que les Dogons ne se placent pas au point de vue de celui qui transmet l'héritage culturel mais au point de vue de celui qui le reçoit. L'accent n'est pas mis sur la «transmission » de l'héritage mais sur sa « réception » : en 
naissant en un lieu on y a «trouvé » ce qui se faisait et se disait ${ }^{37}(1.57,65-67,89,90)$. Dans ce contexte culturel, «trouver » signifie aussi « recevoir » et même si, en pratique, cela consiste à re-dire et re-faire $(1.57-58,65,70)$, la conception dogon de la « réception » laisse de la place à l'invention ${ }^{38}$. Elle autorise une marge de choix et d'interprétation dans ce qui est entendu ou compris $(1.54-58,69,88-90)$ : la « réception » de l'héritage consiste d'abord en une revendication consciente avant d'être une proclamation active. Cette conception de la tradition comme réception d'un héritage pose donc autrement la question de la " conservation ${ }^{39}$ ".

Car l'impression dominante que l'on retire de la lecture du texte est celle d'une réception passive qui ne serait en rien affectée par le présent de la société (1. 30-34). Mais il ne s'agit sans doute que d'une figure de rhétorique destinée à souligner la continuité en marquant ostensiblement la fidélité au message des ancêtres. En effet, bien que les locuteurs ne cessent de répéter qu'ils se conforment à ce qu'ils ont entendu et s'en tiennent à ce qu'ils ont compris, on constate qu'ils s'entourent systématiquement de précautions oratoires leur permettant de ne pas s'engager personnellement sur l'exactitude des énoncés proférés. C'est ainsi que tous demandent aux divinités invoquées d'excuser les erreurs qui pourraient possiblement se glisser dans leurs dires (1. 22, 63), qu'ils attribuent, par avance, à leur ignorance d'enfant ${ }^{40}$. Cette image du "petit-enfant» qui revient fréquemment $(1.22,31,32,66,88)$ met en jeu plusieurs représentations. En premier lieu, la filiation, qui apparaît à la fois comme emblème de la continuité sociale et de la responsabilité hiérarchique: les locuteurs ne sont pas les pères fondateurs des événements, ils ne sont que les descendants héritiers de la situation! (1. 32, 54-57). En second lieu, elle évoque l'ignorance $(1.55,56,58,70,88)$ et l'irresponsabilité de l'enfant qui ne peut que répéter sans avoir toujours bien compris ce qu'il a entendu (1. 54-58, 65, 67 à 70, 89, 90).

L'insistance mise à se présenter comme des enfants irresponsables montre qu'une marge de liberté est prise avec la forme littérale de l'énonciation. Mais cet espace de liberté et de créativité ne préjuge pas du souci de conformité qui reste attaché au contenu: la réception paie sa dette à la transmission qui la rend possible.

Le souci de fidélité se manifeste de plusieurs manières. En premier lieu, la conformité est garantie, de manière interne, par la croyance partagée que toute erreur importante, tout " glissement de la langue " pourrait déclencher l'ire ancestrale ou la colère divine (1. 92, 93,102,137) dont il convient de se prémunir $^{41}$ (1. 105, 110). En revanche, la liberté d'expression dont font preuve les dignitaires religieux tendrait à prouver que le «lien spécial " qu'exprime leur position les protège d'un certain nombre de conséquences désagréables. En second lieu, la conformité de contenu est aussi garantie par la tradition des autres : celle des clans dogons voisins. En effet, il faut garder en mémoire qu'une tradition n'est jamais que celle d'un groupe particulier ${ }^{42}$ : la délimitation de ses marges de variation, d'invention ou d'omission est en quelque sorte garantie, de manière externe, par la tradition des groupes voisins qui ont souvent leur propre version des mêmes événements fondateurs. 


\section{Un contexte « traditionnant » : la parole sous tutelle divine}

18 La procédure d'ouverture du conseil, brève mais très solennelle, marque la rupture avec l'ordre commun du discours et l'entrée dans la proclamation de la tradition où le rapport du discours à son objet change complètement. La condition «traditionnante » majeure est remplie par la désignation solennelle du dignitaire religieux lèbè dugo-no $0^{43}$ comme premier locuteur (1.1). En effet, cette position religieuse présuppose une relation spéciale et exclusive entre, d'une part, son titulaire, le doyen du clan, et d'autre part, la divinité lèbè dont il est le serviteur et les ancêtres dont il est garant. L'autorité du lèbè dugo-no est signalée de plusieurs manières. Elle se manifeste dans le passage de la parole à un cadet (1. 23), dans la préséance qu'il occupe dans l'ordre d'énumération des présents (1. 72) et dans la référence qui est faite à la garantie de sa parole (1. 91). De même, pendant la narration, la distribution de la parole ne doit rien au hasard. Bien au contraire, au fur et à mesure qu'on descend les degrés d'antériorité dans la hiérarchie des divinités $(1.4,5,8,9)$ puis des ancêtres (1. 13, 14, 20, 24, 27, 39, 40), la parole est distribuée proportionnellement au rang d'aînesse décroissant des membres du conseil qui se trouvent présents et en mesure de parler (1.23).

Dans les situations d'interlocution quotidiennes, les ancêtres dogons sont simplement évoqués, mais dans la situation « traditionnante » créée par le conseil du clan, le rapport entre les entités décrites et le discours qui les vise change complètement : il devient un rapport de cause à conséquence ${ }^{44}$. Ce rapport de causalité est au fondement même du « lien spécial » qui s'instaure entre la position de l'énonciateur et le domaine ancestral visé par son discours ${ }^{45}$. Dans le texte, l'instauration de ce lien apparaît dans les changements de style narratif. On remarque en effet que, parfois, le locuteur abandonne le style indirect - qui donne une simple représentation des faits ancestraux (1. 20, 39, 41, $67,68)$ - pour passer au style direct ${ }^{46}$ par lequel l'ancêtre invoqué devient, par la bouche $\mathrm{du}$ locuteur, l'acteur de ce qui est en train de se dire $(1.26,54)$.

Il en va ainsi de l'ancêtre samolu kindê qui est invoqué et chargé de la tutelle de la narration en cours qu'on lui demande, de façon très directe, de conduire (1. 26). Cependant, ce lien spécial se manifeste plus clairement, sans doute, dans l'extraordinaire liberté de parole dont font preuve les dignitaires religieux concernés. Ainsi, ce moment où le doyen impute aux divinités ancestrales la responsabilité de tout ce qui vient d'être dit du passé (1. 54). Et surtout, cette situation extraordinaire (1. 92-123), où le dignitaire religieux kezu accomplit un sacrilège.

21 La mise sous tutelle divine et ancestrale de la proclamation apparaît bien comme la principale procédure «traditionnante» mise en place par le contexte. Remarquons toutefois que si la tradition n'existe que proclamée, le mécanisme de la proclamation ne suffit pas à l'instituer.

\section{Un mode de légitimation de l'identité culturelle, religieuse et politique}

Si le contexte ne suffit pas à instituer la tradition on peut se demander en quoi consiste l'héritage ainsi «traditionné » que l'on accepte de revendiquer comme tradition? Car, à 
l'évidence, ce n'est pas n'importe quel contenu culturel qui traverse ainsi le temps. Si l'on s'en tient à ce que dit le texte, on constate que sont successivement abordées les questions de l'affiliation religieuse du clan, celles de sa continuité politique et de l'histoire du peuplement. On peut dire que « la » tradition est la réponse trouvée dans le passé à la question de l'identité karambé formulée dans le présent. Or, pour n'importe quelle société, quels que soient le lieu ou la période considérés, la question de son identité est fondamentale : «La tradition est notre manière d'être, nos mœurs, ce qui caractérise notre famille, notre religion, notre milieu social, notre nation ${ }^{47}$.»

On peut donc considérer, en première hypothèse, que l'autorité de la tradition réside dans le caractère fondamental (au sens propre du terme) pour l'identité actuelle du groupe concerné, de la «foi » qu'elle professe ${ }^{48}$, de la généalogie qu'elle proclame et des événements historiques qu'elle revendique.

Les usages de la langue permettent de distinguer assez finement trois conceptions de la culture dogon: la culture comme "tradition reçue» dogo atêmbo, la culture au sens général de «l'histoire dogon » dogo soo-go, et la culture comme « voie dogon » dogo ozu. Dans le texte, les divers locuteurs utilisent l'expression soo-go, littéralement « la parole », qui est une variable polysémique évoquant la forme littérale de la tradition : le discours qui est entendu et compris (1.69). Ainsi, soo-go signifie, selon le thème abordé, la « vision du monde » (1. 34), la « tradition religieuse » (1. 60), « l'histoire » (1. 64) ou la « narration de la tradition » (1.85).

Bref, on est renvoyé à cet ensemble plus ou moins cohérent qu'on appelle le patrimoine culturel dont la revendication trace les limites de l'identité locale : ko dogon ozu, « ça, c'est la voie dogon ", entend-on fréquemment. La métaphore de « la voie » ozu ${ }^{49}$ - qui exprime quasi universellement la notion de «système de valeurs et de représentations " - traduit indifféremment la notion de culture ou de religion. La première connotation associée à cette notion de «voie», c'est la «singularité» du système de valeurs et de représentations qui rend toute culture particulière et différente des autres. Ainsi, la pensée dogon ne confond-elle pas «la tradition» avec «la coutume» ozéé ${ }^{50}$, conçue comme une pratique sociale, « une manière de faire » propre à un groupe particulier. Les coutumes locales diffèrent mais toutes les différences ne sont pas des produits traditionnels! C'est d'ailleurs ce qui ressort de cette remarque formulée d'un «traditionniste» dogon: "Dans les différents villages la manière de procéder peut varier. Les racines de la culture dogon sont les mêmes, mais la façon de faire peut varier. Toutes les données d'enquête montrent que ces variantes appartiennent à la même culture ozu $u^{51}$.»

La seconde connotation est la "continuité ». La « voie » évoque une piste longiligne qui commence en arrière (dans le passé de celui qui chemine), qui continue en avant (dans son avenir) et qui permet la «marche » et le "voyage » ozéé : la continuité culturelle est donc à la fois spatiale et temporelle. Cette conception est centrale dans l'expression littérale de la tradition dogon. En effet, le mythe d'origine commun à l'ethnie dogon se rapporte à une migration initiale depuis le Mande (1.130) au cours de laquelle le « cheminement » ozéé des ancêtres défricheurs ${ }^{52}$ et fondateurs $(1.20,24,27,46,48,49,52)$ a ouvert « la voie » ozu à ceux qui suivaient.

Enfin, la dernière connotation rappelle qu'au-delà de toutes les assignations statutaires il subsiste toujours la possibilité, relative certes mais bien réelle, de choisir sa "voie » comme aujourd'hui avec l'adoption massive de la religion musulmane ${ }^{53}$. 
Avec une belle cohérence dans les représentations, la notion de "culture " renvoie à l'ensemble des rites et des pratiques coutumières ${ }^{54}$ ozéé que les Dogons considèrent comme caractéristique de leur culture mais aussi de leur pratique religieuse dogon t $\grave{a}^{55}$. Si l'on considère ce qu'il est convenu d'appeler la religion "traditionnelle", au sens commun du terme, l'ensemble des pratiques cultuelles se distribue classiquement autour des deux grands pôles que sont les obligations rituelles ozee yezee et les interdits religieux dama tà qui délimitent, de part et d'autre, la voie religieuse dogon. Cette voie religieuse singulière apparaît comme un élément central de l'identité ethnique ${ }^{56}$ (1.126-137, 142-144) qui se caractérise par la croyance commune en un Dieu céleste amba (1. 8, 98, $101,129)$, une Déesse chtonnienne yayye ${ }^{57}(1.9,10,128,129)$ et un ancêtre mythique lèbè (1. 13). En revanche, la variété des cultes locaux - comme celui du ba-binu (1. 14, 35-37, 92-145) ou celui adressé aux ancêtres wagum (1. 20, 24, 27, 39, 40) - qui marque les particularismes segmentaires, lignagers ou claniques, est un des éléments qui permettent de comprendre la multiplicité des traditions locales à l'intérieur d'une même société.

La deuxième partie du texte (1. 88-145) - qui rend compte de la palabre ayant suivi la proclamation stricto sensu - nous met de plain-pied dans une situation blasphématoire extraordinaire (1. 92-123). Le blasphème, accompli par le serviteur du culte lui-même (1. 105, 110, 119-123, 138-139), a consisté à dévoiler le «mystère » de la double nature humaine et divine du ba-binu, l'ancêtre commun aux Karambé et à quelques autres clans dogons (1. 111-115).

Les circonstances du blasphème soulignent l'articulation du secret au sacré. Cette articulation, qui fait partie du processus «traditionnant », est normée. Et c'est parce que cette norme est transgressée $(1.94-100,107,108,138,139)$ que les traditionalistes $(1.34$, $60,92,93,101,102,135-137)$ crient au blasphème $(1.103,104,106-109)$ et sanctionnent immédiatement le délit (1. 119-123). Ce faisant, les Dogons karambé nous disent que le contenu de la tradition religieuse est effectivement au « fond de la crypte ». Mais ils nous disent aussi que, conformément à la coutume, l'entrée de celle-ci doit rester cachée aux non initiés (1. 92-102, 106-109, 115-123).

31 La proclamation de la tradition religieuse légitime l'identité ethnique dogon mais aussi, de manière plus segmentaire, l'identité du clan karambé par la délimitation entre le dedans des Karambé et le dehors des « autres » Dogons (1. 126-130,142-144). La tradition «traditionnée » apparaît donc comme un enjeu qui se constitue autour d'une certaine conception traditionaliste de l'identité.

32 Au-delà du culte des ancêtres, les événements fondateurs de l'histoire du peuplement qui sont évoqués à plusieurs reprises, quoique de manière brève $\mathrm{e}^{58}(1.39,41,45-54,67,68)$, ou résumée, nous proposent une certaine conception de l'Histoire. C'est-à-dire, dans ce cas, un régime d'historicité bien connu des ethnologues: le présent de chaque génération consiste, dans certaines circonstances à se saisir, non pas du passé en général, mais de certains événements qui se rapportent précisément à l'origine du groupe et qui, en tant que tels, constituent les fondements historiques locaux de son identité politique actuelle. À cet égard, la grande réticence de la société à constituer les événements fondateurs en phénomènes historiques montre que, si une partie de l'Histoire dogon est effectivement connaissable par l'anthropologue, le savoir historien n'y a pas encore trouvé sa place (1. 30, 34, 54-58).

33 Sur ce plan aussi, le caractère rétrospectif de la tradition est manifeste. D'abord, le petit nombre d'ancêtres évoqués (quatre en tout !) montre, si besoin était, qu'il ne s'agit dans 
ce cas ni de filiation, ni de généalogie, mais bien d'une mémoire sociale sélectivement construite et réaménagée autour de personnages $(1.14,20,24,27,39,40)$ et d'événements (1. 39-53, 66-68) qui sont au cœur des enjeux politiques contemporains ${ }^{59}$. Ces enjeux sont perceptibles dans le contenu de l'énoncé qui proclame que, historiquement, des droits importants, comme ceux associés à la qualité de fondateur revendiquée par les Dogons karambé $(1.39,45-54,67,68)$, ont été établis en pratique et que cette pratique s'est coutumièrement maintenue jusqu'à aujourd'hui (1. 20, 39, 40, 49-54). Ils sont aussi perceptibles dans le fait que le locuteur se présente comme le successeur des ancêtres (1. 20-34) censés avoir joué un rôle essentiel et fondateur dans l'appropriation du territoire et l'autonomie politique du $\operatorname{clan}^{60}(1.13,15,20,35-37)$ et dont l'autorité politique s'est perpétuée jusqu’à présent (1. 30-34).

Quand un Dogon veut attester la légitimité de son pouvoir il dit : «Je ne l'ai pas acheté ! » Ce faisant, il proclame que la position de successeur ne peut s'acquérir car elle est un attribut de l'aînesse assignée par le rang de naissance. En se situant au point de vue de l'en-groupe, conçu comme la communauté de partage d'une tradition, on constate alors que la revendication de l'héritage religieux et de la succession politique suffit, en soi, à signifier la continuité, quels que soient, par ailleurs, l'ancienneté réelle et l'état de conservation de ce qui échoit.

C'est donc moins la tradition qui tend à se perpétuer inchangée qu'une autorité sociale ou religieuse qui cherche à se maintenir (1.30) en revendiquant son origine et sa légitimité dans des événements dont la tradition constitue la mémoire officielle et partagée. C'est pourquoi les variantes et versions de la tradition ne sont pas, a priori, en concurrence les unes avec les autres mais plutôt en contraposition. Ce qui permet aux contemporains de s'affronter par passés interposés, non seulement en choisissant leurs ancêtres, mais surtout en choisissant parmi les événements accomplis, ou subis, par les ancêtres ceux dont il importe de garder la mémoire afin de légitimer les positions de domination historiquement acquises. La tradition est donc aussi un instrument de pouvoir puisqu'elle offre « à ceux qui l'énoncent et la reproduisent au jour le jour le moyen d'affirmer leur différence et, par là même, d'asseoir leur autorité » sur ceux qui s'y soumettent ${ }^{61}$. Car c'est bien aussi de cela qu'il s'agit : la tradition a un contenu éminemment politique ${ }^{62}$. Elle fonde la légitimité des positions d'autorité, ainsi que les privilèges y afférant, structurant le groupe qui en revendique l'héritage.

On est ici très loin d'une conception de la tradition comme savoir général et théorique sur le monde qui serait distinct du savoir ordinaire ${ }^{63}$. On est renvoyé, de façon surprenante, à une conception constructiviste de la culture qui trouve rapidement ses limites dans le fait que toute construction historique porte la marque de sa faiblesse : elle peut être aussi bien défaite par les contemporains qu'elle fut faite par les ancêtres! D'où la volonté, manifeste, de ne pas historiciser les événements fondateurs afin de les enraciner dans une antiquité indéterminée et essayer, ainsi, de naturaliser la «communauté » qui, de ce fait, n'a pas besoin d'autre définition que l'autoproclamation de soi. C'est ainsi que le traditionalisme dogon proclame la continuité par l'invention permanente d'une tradition qui constitue le mode privilégié de légitimation de l'identité religieuse et de l'autorité politique face à d'autres identités et autorités qui s'appuient sur des légitimités considérées, non point tant comme récentes, mais plutôt comme étrangères et extérieures $^{64}$. La continuité de la tradition est celle d'une autoadministration de soi.

Décidément donc, la traditionalité de la société dogon pose problème. En effet, non contente de permettre, dès la période historique précoloniale, le choix de la tradition 
parmi d'autres alternatives possibles comme l'islam, cette culture a, en outre, inventé le concept de tradition ${ }^{65}$, dont l'émergence constitue la deuxième caractéristique nécessaire à l'apparition du traditionalisme. En effet, pour G. Lenclud, «le traditionalisme n'est nullement une attitude traditionnelle. Une société traditionnelle, telle qu'elle est communément caractérisée, ne saurait être traditionaliste ${ }^{66} \%$. La culture dogon seraitelle moderne ? Personnellement, je considère que les sociétés traditionnelles, au sens de sociétés dans lesquelles «d'ordinaire, les hommes ne connaissent pas leur tradition parce qu'ils la vivent ${ }^{67} "$, n'ont jamais existé. Dire cela, c'est considérer la dimension historique des sociétés dites traditionnelles en postulant qu'elles furent toujours, et partout, en situation d'interaction avec des cultures voisines et de ce fait nécessairement confrontées, sur un mode dominant-dominé le plus souvent, aux traditions dont la culture des « Autres » était porteuse.

On voit bien comment le changement fraye son chemin : il suffit simplement de ne pas recevoir ce qui échoit, ne pas refaire ce qui a été fait et ne plus proclamer ce qui a été dit : c'est-à-dire interrompre la continuité en ne revendiquant pas l'héritage. L'ethnologue est évidemment d'accord pour considérer que la question du changement ou de la conservation de la tradition est un faux problème anthropologique. Mais, pour autant, il ne doit pas oublier que cette question demeure un vrai problème "politique " pour l'autorité traditionaliste qui, dans le monde rural, continue à fonder sa légitimité sur des pratiques et des conceptions ${ }^{68}$ qui s'inscrivent, de manière reconnue, dans un rapport de continuité avec l'héritage « traditionné ».

\section{Le néo-traditionalisme urbain}

39 Il est, en revanche, plus surprenant de voir cette volonté de "traditionnement » de l'autorité apparaître aujourd'hui, loin du pays dogon, dans la capitale, où des fonctionnaires et des hommes d'affaires dogons sont engagés dans des stratégies de légitimation «néo-traditionalistes» de leurs pratiques politiques ou économiques. Témoigne de ce phénomène la création récente d'une «Association Malienne pour la Protection et la Promotion de la Culture Dogon » Ginna Dogon ${ }^{69}$. Le néo-traditionalisme qui l'anime est un phénomène, à la fois urbain et récent, qui se distingue du traditionalisme par le fait que ses promoteurs n'adhèrent pas à la tradition: les fondateurs de l'association sont tous des lettrés, dont les familles sont musulmanes ou chrétiennes, souvent depuis plusieurs générations, qui appartiennent aux espaces socio-culturels urbains de la capitale et qui exercent, ou ont exercé, des fonctions économiques, politiques ou administratives au niveau national.

Un des objectifs culturels majeurs de l'association est de travailler à la fabrication d'une version conforme de "la tradition dogon ». Après avoir considéré tout ce que nous en savons, il est impossible d'imaginer qu'il puisse exister « une » tradition dogon, valable pour toutes les fractions dogons, qui serait en mesure d'affirmer quelque chose de plus que le mythe d'origine commun : l'émigration originelle du Mande ${ }^{70}$. L'historien doit donc s'interroger sur le risque que présente une telle entreprise, à savoir que la fabrication de « la » tradition dogon ne pourrait se faire qu'au détriment de la collecte « des » traditions dogons. C'est-à-dire en abandonnant une bonne part de l'héritage historique, la plus riche et variée, pour en promouvoir une autre inévitablement réductrice mais plus homogène et unifiante. Ces craintes ne sont pas tout à fait injustifiées. Ainsi, le projet de création d'une fondation Marcel Griaule - un des objectifs culturels proposés par l'association 
Ginna Dogon - laisse penser que la «bonne version» de la tradition pourrait être déjà écrite: celle, bien sûr, qui a fait le succès international de la culture dogon. Ainsi stabilisée par une écriture reconnue et légitimée par l'association, «la» tradition constituerait une ressource intéressante : un support symbolique à partir duquel pourrait se mettre en place l'exploitation économique de l'espace culturel dogon, qui fait partie des objectifs de développement de l'association. Cette mise en valeur se ferait par les « entrepreneurs ethniques» de la diaspora dogon bamakoise, et pour leur plus grand profit, à partir des ressources artisanales, touristiques et culturelles du pays dogon, aujourd'hui mondialement connues. L'association Ginna Dogon s'est donc constituée autour d'une revendication néo-traditionaliste qui consiste à se faire reconnaître l'autorité de proclamer officiellement «la» tradition dogon - à partir de positions d'énonciation non "traditionnelles ${ }^{71}$ " et décontextualisées ${ }^{72}$ - dans le but de s'en réclamer pour légitimer certains choix économiques ou politiques et informer les réponses contemporaines qu'exige la situation actuelle. La continuité historique et culturelle invoquée par l'association est donc doublement factice.

\section{En conclusion}

41 À la lumière d'une démarche qui cherche où et comment s'élabore cette "réponse, trouvée dans le passé, à une question formulée dans le présent » qu'on appelle tradition ${ }^{73}$, la tradition dogon apparaît comme autre chose que le référent toujours absent d'une posture traditionaliste qui semble, dans cette société tout au moins, fort ancienne. Elle est ainsi "fabriquée » par un contexte "traditionnant", la contextualisation consistant à mettre sous tutelle divine l'énoncé de la tradition proclamé par un locuteur dont la légitimité tient au "lien spécial ${ }^{74}$ » que sa position rituelle entretient avec le domaine divin et ancestral visé par cet énoncé. Le « traditionnement » opère donc, dans ce cas, par transcendance des conditions humaines et conjoncturelles de l'énonciation.

Il va de soi qu'une telle procédure «traditionnante » interdit, normalement, toute mise en question de la validité de l'énoncé. Ce faisant, elle empêche aussi que la société fasse l'expérience de la relativité de ses valeurs et de l'aspect conventionnel de sa tradition. Mais l'accomplissement de cette procédure n'exprime rien d'autre que la puissance et la légitimité locale du système d'autorité traditionaliste qui ne permet pas que tout un chacun joue avec les valeurs et les normes. À cet égard, les circonstances du blasphème témoignent que la tradition sur l'origine des cultes ou la nature des divinités ne peut pas encore être constituée en objet de savoir. Mais elles témoignent aussi que le traditionalisme tolère une certaine marge de jeu sur les normes de la part des responsables de la tradition.

43 En même temps, l'existence de ce savoir «traditionné », gardé secret «comme trésor caché dans la crypte ", donne quelque réalité à la conception de la tradition comme proclamation « d'un contenu de sens... révélé dans sa vérité intrinsèque $\mathrm{e}^{75}$ ». C'est, en particulier, ce contenu de sens qui fonde l'identité religieuse et politique dogon et qui constitue la source de légitimité des positions d'autorité qui s'en réclament et la proclament.

44 C'est pourquoi la tradition ne procède pas seulement d'un échange entre "passé interprété » et "présent interprétant ${ }^{76}$ ", elle procède aussi et surtout d'un mode de légitimation de l'identité et de l'appartenance collective qui s'inscrit dans une dialectique de l'en-groupe et du hors-groupe. Celle-ci passant par la confrontation ${ }^{77}$ des légitimités 
religieuses et politiques endogènes avec celles conçues et vécues comme imposées de et par l'extérieur.

Dans le traditionalisme dogon, on voit bien comment la tradition "traditionnée " constitue sa référence légitimatrice, tandis que la tradition "traditionnante » constitue son mode de légitimation. Cette question centrale de la légitimation place la tradition à la croisée des questions de pouvoir, d'autorité et d'identité. Dès lors, la différence irréductible qui subsiste entre la conception traditionaliste de la tradition - la réception de l'héritage - et sa conception moderniste - l'interposition du jugement critique entre l'héritage et sa réception - ne tient-elle pas au simple fait suivant: la conception moderniste ne peut exister, et donc prédominer, que dans une société où, pour l'essentiel, le traditionalisme n'est plus le principal instrument de légitimation du pouvoir politique, de l'affiliation religieuse et de l'appartenance collective? C'est-à-dire quand, historiquement, la tradition n'est plus légitime $a$ priori.

\section{NOTES}

1. G. Lenclud, "Qu'est-ce que la tradition?», in M. Détienne, ed., Transcrire les mythologies. Tradition, écriture, historicité, Paris, Albin Michel, 1994, p. 25.

2. Cf. la définition qu'en donne J. Pouillon : « La tradition se définit - traditionnellement - comme ce qui d'un passé persiste dans le présent où elle est transmise et demeure agissante et acceptée par ceux qui la reçoivent et qui, à leur tour, au fil des générations la transmettent. » (J. Pouillon, «Tradition», in P. Bonté \& M. Izard, eds, Dictionnaire de l'Ethnologie et de l'Anthropologie, Paris, Presses universitaires de France, 1991, p. 710.)

3. J. Pouillon, «Tradition : transmission ou reconstruction », in J. Pouillon, Fétiches sans fétichisme, Paris, Maspéro, 1975, p. 160.

4. J. Pouillon, «Plus c'est la même chose, plus ça change ", Nouvelle Revue de Psychanalyse, XV, 1977, p. 208.

5. «La tradition est donc une réponse, trouvée dans le passé, à une question formulée dans le présent. » (G. Lenclud, op. cit., p. 33). Si toute tradition est effectivement une rétro-projection, alors la tentative de E. Hobsbawm (E. Hobsbawm \& T. Ranger, eds, The Invention of Tradition, Cambridge, Cambridge University Press, 1983) de marquer, à propos des traditions historiques, une différence entre les «vraies» traditions - qui seraient dotées d'une réelle profondeur temporelle - et les «traditions inventées » - qui forgeraient de toutes pièces leur continuité historique - n'apporte guère, sur ce plan, d'élément nouveau au débat.

6. J. Goody, «Mémoire et apprentissage dans les sociétés sans écriture: la transmission du Bagré », L'Homme, XVII (1), 1977, p. 29-52.

7. E. Weil, Essais et conférences, t. 2, Le Politique, Paris, Vrin, 1991.

8. «La véracité des énoncés traditionnels est fonction de positions d'énonciations fondées sur un rapport causal entre un certain domaine de réalité et le discours qui le vise. Cette hypothèse fait de la vérité une qualité, non des discours ni de leur rapport avec le monde, mais du rapport entre la personne de l'énonciateur et le monde. » (P. Boyer, « Tradition et vérité », L'Homme, XXVI (1-2), 1986, p. 325-326.)

9. Ce clan dogon participe pleinement d'une culture que les constructions savantes, mais néanmoins partiales et partielles, de l'ethnologie classique française ont, à leur manière, 
« inventé » : elles l'ont constitué en archétype d'une société « traditionnelle » ouest-africaine bon à penser pour la société « moderne » européenne.

10. Ces choix conceptuels renvoient aux travaux de G. Lenclud: "Qu'est-ce que la tradition?», op. cit. ; « La tradition n'est plus ce qu'elle était », Terrains, 9, 1987, p. 110-123. Cet article exprime clairement toute la reconnaissance que je lui dois en ce domaine.

11. G. Lenclud, «Qu'est-ce que la tradition?», op. cit.

12. En 1983, je commençais la transcription intégrale et commentée des enregistrements avec, entre autres, la collaboration irremplaçable de S. Karambé qui avait participé au conseil. C'est en m'engageant dans ce travail que j'ai pris toute la mesure du côté paradoxal de la transcription ethnographique qui consiste à « consigner par écrit [...] une oralité consubstantielle à la tradition en respectant autant que faire se peut l'originalité du moyen de transmission autochtone » (G. Lenclud, «La tradition n'est plus... », op. cit., p. 113). Dans ce cas précis, mon respect de l'énoncé original se marquera par de simples évocations puisqu'il m'est ici impossible de présenter l'intégralité de la transcription. Je m'en console avec cette réflexion de G. Lenclud évoquant l'étymologie commune à la tradition et à la traduction: "Bref, les traditions, autant que les traductions, sont de belles infidèles; cela ne nuit en rien à leur réputation. » (G. Lenclud, "Qu'est-ce que la tradition?», op. cit., p. 30).

13. Dans une société de l'oralité, communiquer des informations sur un domaine de réalité donne à penser que l'on a un accès privilégié aux informations utilisées. Et quand l'interlocuteur ne se considère pas comme placé dans cette position (1.93-99, 107, 86 et 119), même s'il sait que l'on sait qu'il sait, il est fréquent d'observer son refus de parler. Il peut cependant manifester sa bonne volonté en disant qui, dans quelle condition, serait en position de répondre. Sur ce point précis, cf. P. Boyer (op. cit., p. 318).

14. Le lèbè dugo-no qui, à ce titre, servait le culte du lèbè c'est-à-dire, à l'époque, A. Karambé du village de Dyombolo.

15. Qui sont à la fois leurs cadets immédiats en termes de parenté et leurs successeurs en termes de pouvoir.

16. La traditionalité de l'énoncé n'est rien d'autre que l'empreinte du procès d'énonciation incluant un ou des locuteurs, des allocutaires, des positions déterminées, un temps, un lieu, un discours qui précède et qui sert de référence (T. Todorov, cité par G. Lenclud, « Qu'est-ce que la tradition? ", op. cit., p. 35).

17. On pourrait m'opposer qu'il ne s'agit pas là d'une situation «authentiquement traditionnelle» puisque la tenue de ce conseil a été motivée par mes questions. Certes! Il convient donc de préciser plusieurs points. Le premier est que le conseil du clan s'est tenu six mois après mon départ en France et donc hors de ma présence. Le second est que les Dogons karambé m'avaient décrit dans les mêmes termes un autre conseil qui avait eu lieu un an ant mon arrivée et qui avait été réuni pour débattre d'un conflit foncier avec un village voisin. Enfin, des traductions d'allocutions cérémonielles tolo et de prières enregistrées en d'autres lieux du pays dogon et par d'autres que moi sur une période couvrant plus d'un demi-siècle témoignent d'un même mode de proclamation (le texte oral est pratiquement identique dans son introduction et sa conclusion) dans des conditions contextuelles très semblables.

18. Cf. par exemple, la «mosquée de Ségou », dans le résumé qui suit la ligne 87, qui évoque une étape de la migration originelle depuis la région du Mande.

19. Une bonne illustration de cette situation nous est offerte par une étude récente portant sur le foncier - A. A. Têmbênê (Tembély), dogo ginE nE taw sO bana kannu. La gestion traditionnelle des terres en pays dogon, Bandiagara, Ginna Dogon (antenne de Bandiagara), 1993, multig. - qui aborde les procédures « traditionnelles » de règlement des conflits fonciers. L'auteur y rapporte qu'après avoir remercié les divinités et les ancêtres, le doyen qui juge le conflit foncier profère des imprécations contre les "adeptes des religions différentes" sênu dên-dên sênun-gombe. Pour le doyen, l'enjeu est celui décrit par E. Weil : «Seul le traditionalisme, affirmons-nous, peut faire 
notre salut, car nous sommes parvenus à un point où nous perdrons notre âme si nous ne retournons pas à ce qui précisément nous a fait ce que nous sommes. » (E. Weil, op. cit., p. 14).

20. Ces dieux d'en haut Al-Amba sont au nombre de sept.

21. Les dieux d'en bas Nya-Amba sont aussi au nombre de sept.

22. Les Dogons partagent la conception très répandue en Afrique de l'Ouest d'un Dieu créateur de l'univers qu'il a ensuite délaissé et confié aux bons soins de ses premières créatures : la déesse terre et les génies...

23. Ancêtre-mythique de référence pour toute l'ethnie dogon.

24. ba-binu : ancêtre totémique, divinité propre à un grand clan dogon.

25. Ancêtre fondateur du clan karambé.

26. L'ancêtre serpent a fondé le territoire et les villages, il a creusé « le nid » et ses descendants les Karambé se contentent d'occuper le nid ancestral.

27. so sên, littéralement "la bonne connaissance »; soo-go signifie " un savoir », " une manière particulière », « un discours », " un langage », " une parole ». Au sens général « un fait culturel, religieux ou historique ».

28. Suit une énumération des noms des villages voisins dans l'ordre de leur installation.

29. ya atembi soo-go soyya " ancienne / la tradition / l'énoncé de / ils ont parlé ».

30. On considère généralement que l'installation des Dogons dans la région actuelle des falaises a eu lieu entre le $\mathrm{XIV}^{\mathrm{e}}$ et le $\mathrm{XVI}^{\mathrm{e}}$ siècle. On ne sait pas combien de temps a duré la migration à partir du Mande, qui est affirmé comme lieu d'origine.

31. Autre manière de désigner Baba A., le doyen qui dirigeait le conseil.

32. Manière de désigner la divinité en cause sans la nommer dans un contexte qui s'annonce délicat.

33. Ce qu'il peut faire parce qu'il est converti à l'islam depuis longtemps déjà.

34. L'auteur de la transgression : kezu M.

35. Pour décider du sens des mots j'ai adopté une méthode de saturation progressive de la signification. De ce point de vue la signification d'un mot est conçue comme une variable dépendante du contexte d'énonciation. La valeur qu'on assigne à cette variable tient compte des conventions de la langue et de l'intention du locuteur, tout en n'oubliant pas que les conventions de sens émergent de l'usage! Saturer la signification d'un mot est une méthode qui consiste à déterminer contextuellement les valeurs sémantiques de toutes les variables repérées au cours des différentes enquêtes.

36. «L'héritage culturel » atembu n'a, bien sûr, rien à voir avec l'héritage au sens économique du terme qui se dit gálu. D'ailleurs ce dernier, ne se « trouve » pas, il « se ramasse » bala.

37. Ces traits qui sont caractéristiques d'un mode de transmission oral toujours en usage localement réfèrent à une conception de la pédagogie par répétition selon laquelle il suffit de dire ou faire pour transmettre et de redire ou refaire pour montrer qu'on a reçu.

38. Parler d'invention de la tradition «a pour fonction de substituer au caractère passif de la tradition conservée la dimension active de la tradition recréée. L'investigation se détourne de l'énigmatique efficience d'un passé « subi » pour se concentrer sur la réception "opérée » de ce même passé » (G. Lenclud, « Qu'est-ce que la tradition?», op. cit., p. 33).

39. À ce propos, voir l'importante et intéressante discussion de la notion de «modèle » et de «version » par J. Pouillon («Plus c'est la même chose... », op. cit.) et reprise par G. Lenclud (« La tradition n'est plus...», op. cit., p.113-114). Voir aussi la mise en cause du principe « d'invariance » par E. Hobsbawm (op. cit.) et celle du principe de «conservation » par P. Boyer (op. cit., p. 313).

40. II est difficile de trouver meilleur exemple de "procès de reconnaissance en paternité » (G. Lenclud, ibid., p. 118).

41. L. 43, 44. Cette assertion n'évoque pas la possibilité d'apparition d'une dispute entre les participants au conseil, mais celle d'une sanction (sous la forme de rancune ou de maladie qui 
apparaîtrait parmi les participants) envoyée par une divinité ou un ancêtre pour sanctionner une mauvaise énonciation de la tradition. Lors d'une autre situation traditionnelle (rite aux Wagum, cérémonie du Bîê, Sibi-Sibi, 1983), le locuteur se protégeait en prenant la peine d'évoquer les ancêtres qu'il pouvait avoir oubliés : "Nos ancêtres, vous tous, ceux qui ont été appelés et ceux qui n'ont pas été appelés, nous les avons priés. »

42. C'est bien pourquoi il semble impossible de pouvoir parler au singulier de «la » tradition dogon.

43. D'autres circonstances rituelles, sociales ou politiques appellent la participation de narrateurs différents, dont la légitimité est fondée sur l'occupation d'une position d'autorité en rapport avec le domaine de réalité considéré. Il en est ainsi du tolo tolo-no qui, pour toute une région, est seul habilité à déclamer la tradition historique du clan tolo lors de funérailles importantes. Il en va de même pour les griots. Ainsi, en mai-juin 1995 a eu lieu la cérémonie de levée de deuil de Bonzon Areta Togo (village de Sadia-Kanda) qui était le doyen des griots de la plaine de Seno. Elle a pour but de rafraîchir et d'harmoniser la mémoire de l'ensemble des griots de la plaine qui se sont rassemblés par secteurs pour cette occasion. (E. Huysecom, communication personnelle.)

44. P. Boyer, op. cit., p. 323.

45. Cf. note 8, p. 97.

46. Ce procédé qui apparaît dans plusieurs phases narratives a, malheureusement, disparu des résumés plus que succints qui subsistent ici.

47. E. Weil., op. cit., p. 9.

48. Le rapport étroit entre la tradition, la religion et l'identité est attesté historiquement par le fait que dans la sous-région, la conversion à l'islam s'est souvent marquée par un changement d'ethnonyme ou de patronyme lignager.

49. ozu nê kundo signifie « indiquer la direction à prendre » tandis que ozu kundo signifie « donner une bonne éducation »: il est amusant de retrouver en dogon la métaphore morale du "droit chemin ».

50. E. Hobsbawm (op. cit., p. 3) a posé le problème que soulève le rapport entre les notions de «tradition» et de « coutume ». Pour lui la coutume est ce qui se fait en pratique et la tradition est dans le formalisme des objets et des attitudes associés à cette pratique. Selon lui, «l'invariance» caractérise la notion de «tradition» alors que les «coutumes» sont, au contraire, ouvertes au changement et à l'innovation tant qu'elles apparaissent compatibles avec ce qui a précédé. «La coutume ne peut se permettre d'être invariante parce que même dans les sociétés «traditionnelles» la vie ne l'est pas.» (p. 2). Une décennie plus tard, T. Ranger devait critiquer sa propre conception de l'« invention de la tradition» (T. Ranger, «The Invention of Tradition Revisited, The Case of Colonial Africa », in T. Ranger \& M. Vaughan, eds, Legitimacy and the State in Twentieth-Century Africa. Essays in Honor of A. H. M. Kirk-Green, Londres, Macmillan, 1993, p. 62-112). J. Pouillon («Plus c'est la même chose...», op. cit.) définit, quant à lui, la coutume comme "une manière de faire établie ». Cette dernière définition correspond littéralement à celle que donnent les Dogons de la coutume.

51. Têmbênê, op. cit., p. 4-5.

52. L'histoire du peuplement des villages abonde en récits où les ancêtres fondateurs sont des chasseurs-défricheurs qui constituent l'avant-garde d'une migration plus importante.

53. sên-sênun ozu « la religion musulmane ». À propos de conversion religieuse, J. Pouillon (« Plus c'est la même chose... », op. cit.) et G. Lenclud («La tradition n'est plus... », op. cit.) ont bien montré que l'estimation d'un taux de transformation ou de conservation de la religion traditionnelle est absurde, de même qu'est dénuée de sens la fixation d'un seuil qui marquerait la limite entre la permanence et le changement.

54. En effet, ozéé désigne un rite ou une pratique coutumière, c'est-à-dire une manière de faire, réglée ou normée, par laquelle s'exprime et s'impose la culture. 
55. tà désigne la pratique religieuse et singulièrement "l'évitement des interdits liés au sacré » dama tà.

56. J. Bouju, «Qu'est-ce que l'“ethnie" dogon?", Cahiers des Sciences humaines, XXXI (2), 1995, p. 329-363.

57. Appelée Isubay lêbê dans les autres dialectes dogons.

58. Pour toutes les questions d'histoire ancienne dont la mémoire risquait de rappeler des actes honteux ou de réveiller de vieilles rancunes dangereuses pour la paix sociale, le locuteur évoque brièvement les faits sans les développer. Il est pris dans un double bind qui consiste, tout à la fois, à dire certaines choses mais, autant que possible, sans les faire savoir ! (1. 86).

59. «Le traditionalisme est un moyen, quelquefois efficace, de lutter contre un changement spécifique, de forme et de contenu déterminés. » (E. Weil, op. cit., p. 16.)

60. J. Bouju, «Fondation et territorialité. Instauration et contrôle rituel des frontières (Dogon karambé, Mali) », in J.-F Vincent, D. Dory \& R. Verdier, eds, La construction religieuse du territoire, Paris, L'Harmattan, 1995, p. 352-365.

61. G. Lenclud, « La tradition n'est plus... », op. cit., p. 119.

62. «Le traditionalisme, dans ce cas, se révèle non point, en dépit de sa prétention, comme la théorie d'un mode de vie en parfaite harmonie avec celui de nos pères, mais comme un instrument utile pour influencer les décisions politiques concernant l'avenir.» (E. Weil, op. cit., p. 16).

63. P. Boyer, Tradition as Truth and Communication : a Cognitive Description of Traditional Discourse, Cambridge, Cambridge University Press, 1990.

64. "Nous ne pouvons pas faire certaines choses, nous ne pouvons pas admettre qu'elles soient faites, si nous ne voulons pas trahir notre être même. » (E. Weil, op. cit., p. 18).

65. «Ces civilisations que nous appelons "traditionnelles" n'admettent pas la moindre possibilité de changement, parce qu'elles ne font aucune place au concept de tradition. » (ibid., p. 13).

66. G. Lenclud, «Qu'est-ce que la tradition?», op. cit., p. 38.

67. E. Weil, op. cit., p. 9-10. Pour P. Boyer, « la tradition en tant que propriété caractéristique d'un objet culturel est un type de rapport cognitif. Là où ce rapport se perpétue, on a une culture traditionnelle et là où il a disparu pour être remplacé par l'autre type, il n'y plus que du traditionalisme ». Le terrain offre des situations plus complexes: on y trouve des gens qui agissent en se situant dans le rapport cognitif de type traditionnel tout en étant en interaction quotidienne avec d'autres qui se situent dans l'autre type de rapport. Tous sont parfaitement conscients des alternatives possibles.

68. Parmi celles-ci le postulat du « lien spécial » formulé par P. Boyer occupe une place de choix. 69. Cette association, créée en 1992 à Bamako (Mali) est non gouvernementale, non confessionnelle, apolitique, à but non lucratif... Elle est aussi ouverte aux Dogons et «dogonphiles » sans distinction de race, d'ethnie, de nationalité ou de sexe, ainsi qu'à toute personne physique ou morale qui adhère aux statuts et règlement intérieur (art. 2, titre I des statuts). À la suite d'E. Hobsbawm (op. cit., p. 8) on doit considérer l'apparition d'un mouvement pour la préservation de la tradition comme symptomatique du néo-traditionalisme urbain. Mais, avec les Dogons, on court le risque de voir le "pays dogon " (déjà classé comme patrimoine mondial par I'UNESCO) transformé en sanctuaire humain naturel destiné à abriter des formes isolées de vie pseudo-archaïque.

70. Dont la transcription tient, en moyenne, en une page de texte! Par ailleurs, d'autres peuples au Mali et au Burkina-Faso (Samo, Dafing...) ont le même mythe d'origine Mande sans partager, en rien, l'identité dogon...

71. Contrairement au traditionaliste, le néo-traditionaliste ne peut pas prétendre occuper la position d'héritier de la «tradition » car il est situé, par définition, en dehors de la sphère de légitimité « traditionnelle». 
72. La conversion à l'islam à la fois ancienne et en progression constante et, de manière beaucoup moins ancienne et massive, au christianisme ont en quelque sorte rendu profanes (profanés ?) les éléments païens de la culture dogon. À Bamako, ces éléments "traditionnels ", qui ne fondent plus la croyance dominante ni la structure d'autorité, n'en constituent pas moins une ressource symbolique qui se trouve disponible pour une utilisation idéologique et économique nouvelle. Voir à ce sujet E. Hobsbawm (op. cit.).

73. G. Lenclud, «Qu'est-ce que la tradition?», op. cit., p. 33.

74. P. Boyer, op. cit.

75. G. Lenclud, ibid.

76. P. Ricœur cité par G. Lenclud, ibid., p. 33.

77. Dans le cadre de cette confrontation, on observe généralement que les structures d'autorité politique, religieuse ou économique endogènes sont plutôt traditionalistes, tandis que les groupes dominés par ces mêmes structures (par exemple, la classe des captifs, l'ordre des castes, ou les villages politiquement dominés aux marches du territoire) trouvent plutôt intéressant d'adhérer à une autre tradition. De même, j'ai pu montrer ailleurs (J. Bouju, Graine de l'homme, enfant du mil, Paris, Société d'ethnographie, 1984) que la position dominée des cadets dans la structure de répartition des biens et dans le système d'héritage était la cause de leur émigration temporaire qui entraîne systématiquement la conversion à l'islam et l'inscription dans les rapports marchands.

\section{RÉSUMÉS}

L'objet de l'article est d'analyser, à partir de la transcription d'un document ethnographique original, la conception dogon de la tradition telle qu'elle est produite par le texte et le contexte. L'analyse, qui met en évidence la primauté de la réception sur la transmission, montre comment la tradition est "fabriquée " par une procédure "traditionnante " qui consiste à placer sa proclamation sous tutelle divine et ancestrale afin, entre autre, d'en garantir la conformité. Cette procédure de «traditionnement " par transcendance constitue la source et le mode privilégiés de légitimation de l'affiliation religieuse et de l'autorité politique «traditionnelles » locales qui sont, depuis longtemps, confrontées à d'autres conceptions religieuses et dominées par des pouvoirs politiques exogènes. Phénomène rural, le traditionalisme dogon proclame la continuité culturelle tout en s'inscrivant historiquement dans une dialectique de l'en-groupe et du hors-groupe. Par contre, le néo-traditionalisme est un phénomène urbain qu'on peut distinguer du traditionalisme par le fait que ses promoteurs - qui sont scolarisés, musulmans ou chrétiens et qui exercent ou ont exercé des fonctions politiques ou administratives au niveau national - se sont récemment « convertis » à la tradition. Ce mouvement néo-traditionaliste tente d'homogénéiser les traditions dogons pour fabriquer une tradition « ethnique » unique susceptible, lui semble-t-il, de légitimer certains choix et d'informer les réponses économiques et politiques contemporaines qu'exige le processus actuel de décentralisation politique de l'État.

The object of the article is to analyze the dogon concept of tradition as it is produced by text and context on the basis of the transcription of an original enthnographic document. The analysis, which reveals the primacy of reception over transmission, shows how tradition is "produced" by a "traditioning" procedure which consiste of placing its declaration under divine and ancestral protection in order to, among other things, guarantee its conformity. This process of 
"traditionment" by transcendence constitutes the source and privileged mode of legitimation of religious affiliation and of local "traditional" political authorities which are, since a long time, confronted with other religious conceptions and dominated by exageneous political powers. A rural phenomenon, dogon traditionalism proclaims cultural continuity registering itself historically at the same time in a dialectic of the in-group and the out-group. On the contrary, neo-traditionalism is an urban phenomenon which can be distinguished from traditionalism by the fact that its promoters - who are educated musulmans or christians and who exercise or have exercised political or administrative functions at a national level - have recently converted to tradition. This neo-traditionalist movement tries to homogenize the dogon traditions to produce a single ethnie tradition, apparently susceptible of legitimizing certain choices and providing contemporary economic and political answers which are required by the actual political decentralization process of the State. 